\title{
Incidental Giardiasis Diagnosed by Fine-Needle Aspiration of a Phantom Cecal "Mass"
}

Dear Dr. Bedrossian:

Differential diagnosis of a submucosal cecal mass discovered by colonoscopy includes neoplasms such as carcinoid, lymphoma, leiomyoma, gastrointestinal (GI) stromal tumor, and lipoma, among others. However, infectious processes such as tuberculosis, actinomycosis, and ameboma in the ileal-cecal regions have been reported presenting as masses mimicking tumors. ${ }^{1-4}$ Giardia is the most common intestinal pathogen worldwide and is the most common cause of protozoal GI disease in North America, producing significant but not life-threatening GI distress and diarrhea. ${ }^{5}$ The trophozoites characteristically localize in the proximal small intestine (in one study: duodenal $82.5 \%$, ileal $12 \%$, gastric $8.7 \%$, jejunal $2 \%$, and colon $0.4 \%){ }^{6}$ Diagnosis is most reliable on morphologic identification of trophozoites and/or cysts in fecal specimen. Identification of trophozoites (pear-shaped body with cilia, two central nuclei, and median body) by examining gastric/duodenal contents or biopsies may be necessary when stool examination result is negative. ${ }^{5,7}$ Although diagnosis of giardiasis may be challenging, treatment is usually successful with antibiotics. ${ }^{8}$ There have been rare occasions when giardiasis was diagnosed by fine-needle aspiration (FNA) of masses in the abdomen; one such case coexisted with neuroblastoma ${ }^{9,10}$ and another was associated with tuberculosis. ${ }^{4}$ In other instances, Giardia was seen in cytologic specimens from peritoneal fluid, bronchoalveolar lavage, and biliary drainage. ${ }^{11-13} \mathrm{We}$ report an interesting case in which incidental Giardia lamblia was diagnosed by FNA procedure performed on a phantom cecal mass.

A 58-yr-old man presented to Johns Hopkins Medical Institutions for a second opinion regarding a "cecal mass" identified by screening colonoscopy at another institution. According to the outside report, a lesion was found in the

*Correspondence to: Robert T. Pu, M.D., Ph.D., Department of Pathology, University of Michigan, 1500 E. Medical Center Drive, Room 2G332/Box 0054, Ann Arbor, MI, 48109. E-mail: robertpu@umich.edu Received 7 February 2005; Accepted 20 April 2005

DOI 10.1002/dc.20351

Published online in Wiley InterScience (www.interscience.wiley.com). cecum, where the appendix is normally located. It appeared to be a submucosal polyp or other lesion with normal overlying mucosa, by colonoscopy. It could not be reduced into the appendiceal opening. Biopsy was taken at the outside hospital and revealed a hyperplastic polyp. On admission to Johns Hopkins Hospital, the patient was found to be in excellent health and not on any medication. There was no family history of GI disease, no blood in the stool, and no weight loss or recent change in bowel habits. There was no other significant medical history. The clinical differential diagnosis included leiomyoma, leiomyosarcoma, carcinoid tumor, lipoma, and retroverted appendix. Endoscopic ultrasound-guided fine needle aspiration (EUS-FNA) was performed. During the EUS-FNA procedure, the mass was observed as a cecal submucosal hyperechoic lesion measuring $6.6 \mathrm{~mm}$. Further observation revealed that the bulge fluctuated and, at times, disappeared. The findings were consistent with a retroverted appendix. At on-site evaluation, several air-dried smears were stained with Diff-Quik ${ }^{\circledR}$ and microscopically examined. Findings included mucus and intestinal columnar epithelial cells admixed with lymphocytes, cellular debris, and bacteria (Fig. 1A). The initial impression was "Negative for malignancy." After processing and examining all slides, closer observation at low power revealed structures that could easily be mistaken for lymphocytes (Fig. 1C). But at higher power, numerous pear-shaped microorganisms with visible central bar body, binuclei, and several cilia, most prominent at the tail end of the organism, were recognized, diagnostic of Giardia lamblia (Fig. 1B and D). Cytologic diagnosis was made as "Negative for malignant neoplasm, presence of microorganisms consistent with Giardia lamblia." The patient was discharged with a prescription for a 2-wk course of flagyl.

This case illustrates the importance of EUS-FNA and correlation of radiological and pathological findings. The incidental finding of giardiasis was made by FNA performed on a cecal "mass" that turned out to be a retroverted appendix. The presence of Giardia trophozoites in this case apparently did not cause any clinical symptoms, as the patient was healthy and without complaints. Because 


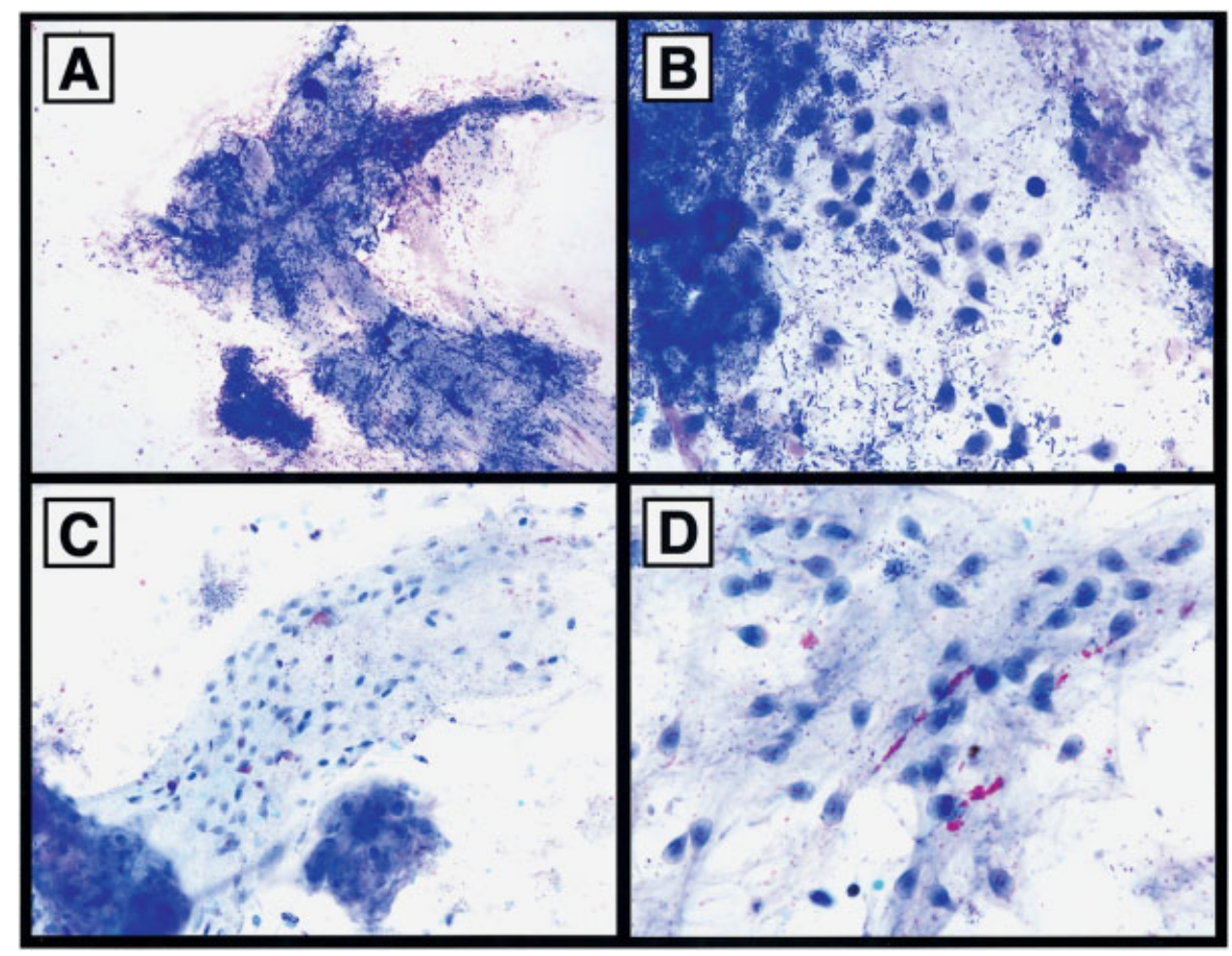

Fig. 1. Giardia lamblia observed with Diff-Quik $^{\circledR}$ (A and B) and Papanicolaou (C and D) staining. At low power view $(A, \times 40$ and $C, \times 200)$, obscured by debris, mucus, and bacteria, Giardia trophozoites were not easily appreciated. At high power view (B and D, $\times 400$ ), typical pearshaped trophozoites diagnostic of Giardia lamblia are easily appreciated. [Color figure can be viewed in the online issue, which is available at www.interscience.wiley.com.]
Giardia is one of the most prevalent protozoans in the human GI tract, its identification was not unusual. However, discovery as a mass, presenting for evaluation by the cytopathology FNA service, was unexpected. As EUS-FNA becomes a more widely used procedure, a mass in the GI tract is often subjected to FNA for initial diagnosis. Most often, a mass represents a neoplastic process. In such cases, on-site triage of FNA by the cytopathologist for ancillary tests, such as flow cytometry for cases suspicious for lymphoma, becomes necessary and essential for making a correct diagnosis. However, reports from others suggest infection by different microorganisms could be responsible for imaging findings of a mass. ${ }^{1,3,4}$ In our case, EUS-FNA provided real time observation of a rare situation in which the mass fluctuated and disappeared, consistent with a retroverted appendix that was initially mistaken for a submucosal mass, although it is not certain whether Giardia trophozoites contributed to the impression of a mass or whether they were just attached to the mucosal surface.

Robert T. Pu, M.D., Ph.D.*
Department of Pathology
University of Michigan
Ann Arbor, Michigan
Dorothy L. Rosenthal, M.D.
Department of Pathology
Johns Hopkins Medical Institutions
Baltimore, Maryland

\section{References}

1. Majeed SK, Ghazanfar A, Ashraf J. Caecal amoeboma simulating malignant neoplasia, ileocaecal tuberculosis and Crohn's disease. J Coll Physicians Surg Pak 2003;13:116-117.

2. Kelly J, Warren K, Coutts M, et al. An unusual case of ileocaecal tuberculosis in an 80-year-old Caucasian male. Int $\mathrm{J}$ Clin Pract 1999;53:77-79.

3. Ferrari TC, Couto CA, Murta-Oliveira C, et al. Actinomycosis of the colon: a rare form of presentation. Scand J Gastroenterol 2000; 35:108-109.

4. Gera N, Singh N, Bhatia A. Unique association of tuberculous enteritis and Giardia lamblia. Acta Cytol 1996;40:389-390.

5. Juckett G. Intestinal protozoa. Am Fam Physician 1996;53:25072518.

6. Oberhuber G, Kastner N, Stolte M. Giardiasis: a histologic analysis of 567 cases. Scand J Gastroenterol 1997;32:48-51.

7. Marshall JB, Kelley DH, Vogele KA. Giardiasis: diagnosis by endoscopic brush cytology of the duodenum. Am J Gastroenterol 1984; 79:517-519.

8. Gupta YK, Gupta M, Aneja S, et al. Current drug therapy of protozoal diarrhoea. Indian J Pediatr 2004;71:55-58.

9. Das DK, Sarin YK, Grover RK, et al. Neuroblastoma with concomitant giardiasis: report of a case with diagnosis by fine needle aspiration cytology. Acta Cytol 2001;45:740-744.

10. Saran RK, Gupta SK, Nijhawan R, et al. Giardia lamblia presenting as a right iliac fossa mass. Acta Cytol 2001;45:280-282.

11. Bloch T, Davis TE, Jr, Schwenk GR, Jr. Giardia lamblia in peritoneal fluid. Acta Cytol 1987;31:783-784.

12. Stevens WJ, Vermeire PA. Giardia lamblia in bronchoalveolar lavage fluid. Thorax 1981;36:875.

13. Perez-Martin G, Gomez-Cerezo J, Codoceo R, et al. Bilirubinate granules: main pathologic bile component in patients with idiopathic acute pancreatitis. Am J Gastroenterol 1998;93:360362 . 